\title{
An Evaluation of Plume Tracking as a Strategy for Gas Source Localization in Turbulent Wind Flows
}

\author{
Javier Monroy \\ MAPIR research group \\ University of Malaga \\ IBIMA biomedical research institute \\ Malaga, Spain \\ jgmonroy@uma.es
}

\author{
Jose-Raul Ruiz-Sarmiento \\ MAPIR research group \\ University of Malaga \\ IBIMA biomedical research institute \\ Malaga, Spain \\ jotaraul@uma.es
}

\author{
Javier Gonzalez-Jimenez \\ MAPIR research group \\ University of Malaga \\ IBIMA biomedical research institute \\ Malaga, Spain \\ javiergonzalez@uma.es
}

\begin{abstract}
Gas source localization is likely the most direct application of a mobile robot endowed with gas sensing capabilities. Multiple algorithms have been proposed to locate the gas source within a known environment, ranging from bio-inspired to probabilistic ones. However, their application to real-world conditions still remains a major issue due to the great difficulties those scenarios bring, among others, the common presence of obstacles which hamper the movement of the robot and notably increase the complexity of the gas dispersion. In this work, we consider a plume tracking algorithm based on the well-known silkworm moth strategy and analyze its performance when facing different realistic environments characterized by the presence of obstacles and turbulent wind flows. We rely on computational fluid dynamics and the open source gas dispersion simulator GADEN to generate realistic gas distributions in scenarios where the presence of obstacles breaks down the ideal downwind plume. We first propose some modifications to the original silkworm moth algorithm in order to deal with the presence of obstacles in the environment (avoiding collisions) and then analyze its performance within four challenging environments.
\end{abstract}

Index Terms-gas source localization, plume tracking, surgecast, mobile robot, robotics olfaction, electronic nose, gas sensor, MOX, turbulent wind flow

\section{INTRODUCTION}

At medium Reynolds values, odor dispersal is dominated by the carriage of the gas molecules by the background fluid currents (i.e., advection), causing an odor plume to appear in the down-flow direction of the source [1]. This phenomenon has led researchers to propose multiple algorithms that track back the source location by navigating through the chemical plume, commonly referred as plume tracking strategies. Research on plume tracking robots started with pure chemotactic approaches, exploiting the chemical gradient within the plume to move towards the source. Proposed strategies include Braitenberg approaches [2] or algorithms based on E. coli bacteria [3], to cite some. Performance was then improved by combining chemotaxis with anemotaxis, exploiting the strong directional cue that the flow direction brings when acting under turbulent flows [4]. Proposed methods include the dung beetle or zigzag method, which involves moving upwind within the

This work has been funded by the Spanish Government (project DPI201784827-R) and the Andalusia Government (project TEP2012-530), both with funds from the European Union (FEDER).
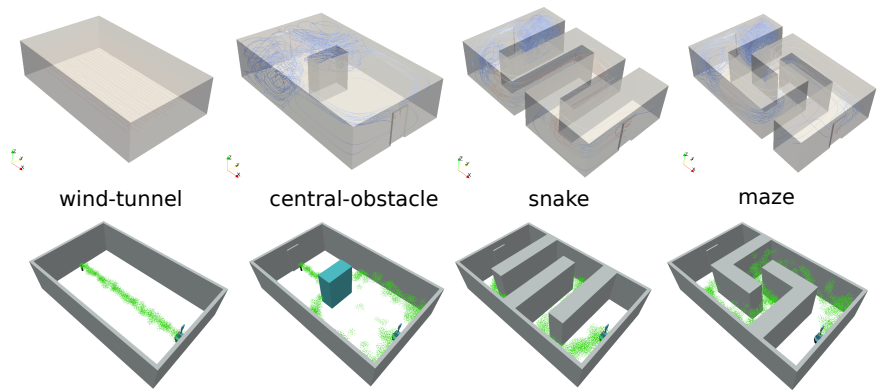

maze

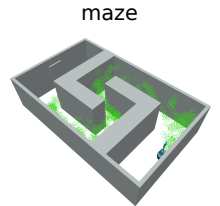

Fig. 1. The four scenarios employed to test plume tracking. (top) CAD models and illustration of the wind flows generated with CFD tools. (bottom) Snapshots of the gas dispersion plumes obtained with GADEN. As can be seen, turbulent flows are predominant in all scenarios except for the wind-tunnel scenario (considered for comparison), leading to patchy and meandering plumes more challenging to track.

odor plume in a zigzagging fashion [5], the plume-center upwind search [6] or the silkworm moth strategy, also known as the surge-cast algorithm [7].

Most of the plume tracking strategies proposed so far have been validated and demonstrated to work under quasi-ideal environmental conditions (i.e., in a laminar wind field where a straight downwind plume is formed from the source location). Yet, as reported by [8], the main drawback of these strategies arises when facing realistic, turbulent environments. Under this challenging environmental conditions, the plume is not straight and continuous, but given the time-varying nature of flow fields and the predominance of turbulence over diffusion, plumes tend to meander, become patchy and, to a far lesser extent, spread out [4]. Furthermore, the plume structure can change over time if the direction of air flow shifts considerably, being difficult to accurately determining the wind flow direction. In addition, the presence of obstacles in the environment will not only negatively influence the performance, but require, in many cases, to substantially modify the algorithm [9].

In this work we present a practical evaluation of a plume tracking algorithm based on the surge-cast method [7], under different and challenging environments characterized by the presence of obstacles, turbulent air flows and non-ideal gas plumes. To do so, we employ computational fluid dynamics (CFD) tools to generate realistic and dynamic wind flows, 


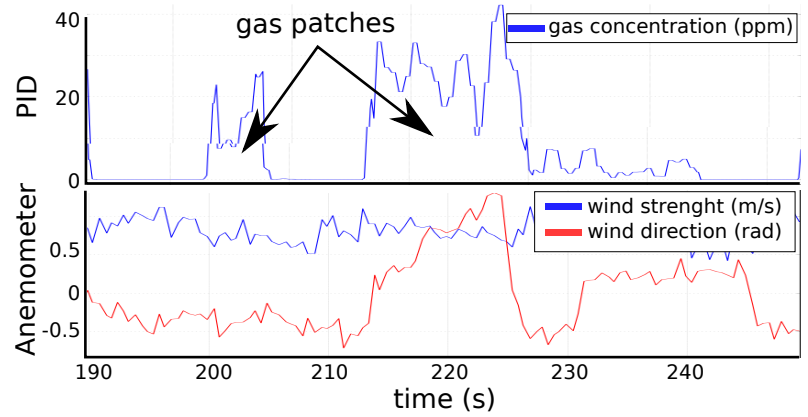

Fig. 2. PID and anemometer simulated measurements recorded during one of the experimental runs. As can be seen, measurements are affected by noise and illustrate the fact that, sometimes, the gas dispersion becomes peaky.

and the open-source gas simulator GADEN [10] to integrate the $3 \mathrm{D}$ and realistic gas dispersion into ROS mobile robotics framework. We first describe the four scenarios employed for simulating the gas dispersal and then outline the plume tracking algorithm employed to locate the gas source. Finally, we present the results and discuss them before providing some future work.

\section{EXPERIMENTAL SETUP}

In this work we consider four different scenarios based on a [10x6x2.5]m arena. These scenarios have been designed to increase the complexity of the wind flows (by introducing new obstacles) and therefore to recreate situations where the robot must confront non-straight and patchy gas plumes. Fig. 1 (top) shows the different scenarios where we have superimposed a snapshot of the turbulent wind flows generated with CFD tools, illustrating the influence of obstacles.

To generate the gas dispersion we employ the open-source gas simulator GADEN [10]. We set the source location at the left-center inlet at $0.5 \mathrm{~m}$ from the floor, releasing ethanol at a constant rate, and set the initial robot location within a circle (of radius $1 \mathrm{~m}$ ) centered at the right-center outlet to increase variability in the results. We simulate the readings of a 2Danemometer and a PID (photo-ionization gas detector) both carried by the robot and located at a height of $0.5 \mathrm{~m}$ from the floor. Fig. 1(bottom) shows snapshots of the different "plumes" generated for each scenario. It can be seen how the straight and ideal plume generated downwind in the wind-tunnel scenario is completely disrupted when introducing obstacles. It must be stressed that despite being simulated data, we are accounting for time-varying and turbulent gas plumes as reflected in the measurements of both sensors shown in Fig. 2.

\section{ALGORITHM}

The plume tracking strategy used here is based on the surgecast algorithm [7] and is implemented as a finite state machine. To deal with obstacles, we enhanced it by considering the navigation capabilities of the ROS architecture, that is, both path planning and reactive navigation based on sensing the robot surroundings. The latter allows us to discard navigation goals that either fall withing obstacles or that are to close to them, avoiding collisions. Moreover, to face the challenging

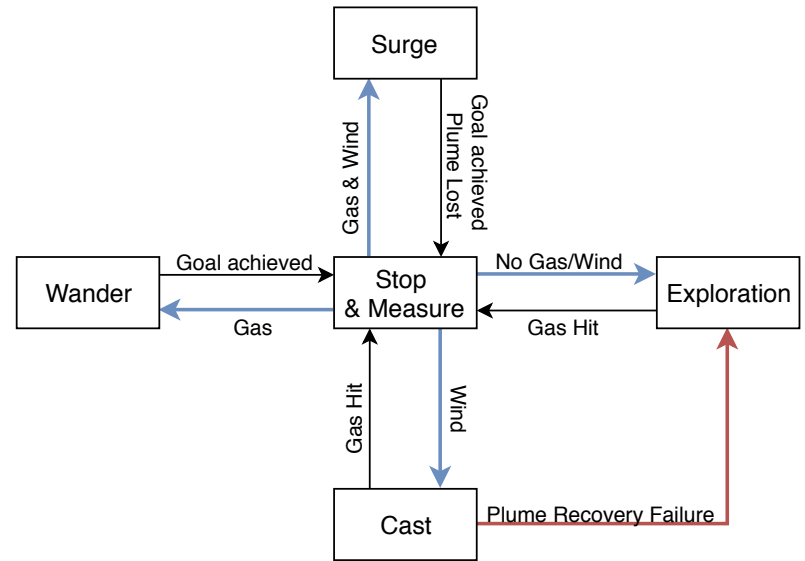

Fig. 3. Block diagram of the plume tracking algorithm depicting the five search states and the conditions for changing to another state.

conditions imposed by turbulent environments we consider new states to deal with situations where either the wind flow is to weak to be measured by an anemometer or where the gas concentration falls bellow the detection threshold, proposing new navigation strategies that may allow the robot to fulfill the search.

Fig. 3 shows a block diagram illustrating the different states and the algorithm flow according to the measurements obtained during the search. To the conventional surge (upwind movement under the presence of the plume) and cast stages (cross-wind movements to recover the plume once it is lost), we introduce an exploration stage to search for gas and wind clues, proposing random navigation goals in the environment, as well as a wander stage aimed to deal with situations where due to the obstacles configuration the robot cannot get a reliable measurement of the wind-flow though gas has been detected. In these situations the robot will wander around until wind is sensed. Additionally, to deal with the dynamic nature of the gas dispersion, we consider a stop\&measure stage where the robot remains still while taking gas and wind observations and consider the average of the these values when changing to a new state.

To asses the performance we use three metrics: the distance and search-time overheads, calculated as the traveled distance or overall search-time divided by the ideal case where the robot navigates directly to the source (estimated by a path planning algorithm that accounts for the obstacles and the robot shape), and the success rate. For the latter we consider a fixed robot starting point and set a maximum search time of $t_{\max }$ (estimated for each environment as the average time a mobile robot would employ in performing a complete sweep of the arena, that is generating a gas distribution map [11]). Experiments are considered successful when the robot reaches a location in the physical vicinity of the source before $t_{\max }$.

\section{RESULTS AND DISCUSSION}

Evaluation of the proposed plume tracking algorithm has been performed by executing the algorithm ten times for each scenario. Moreover, we consider two wind flow conditions 

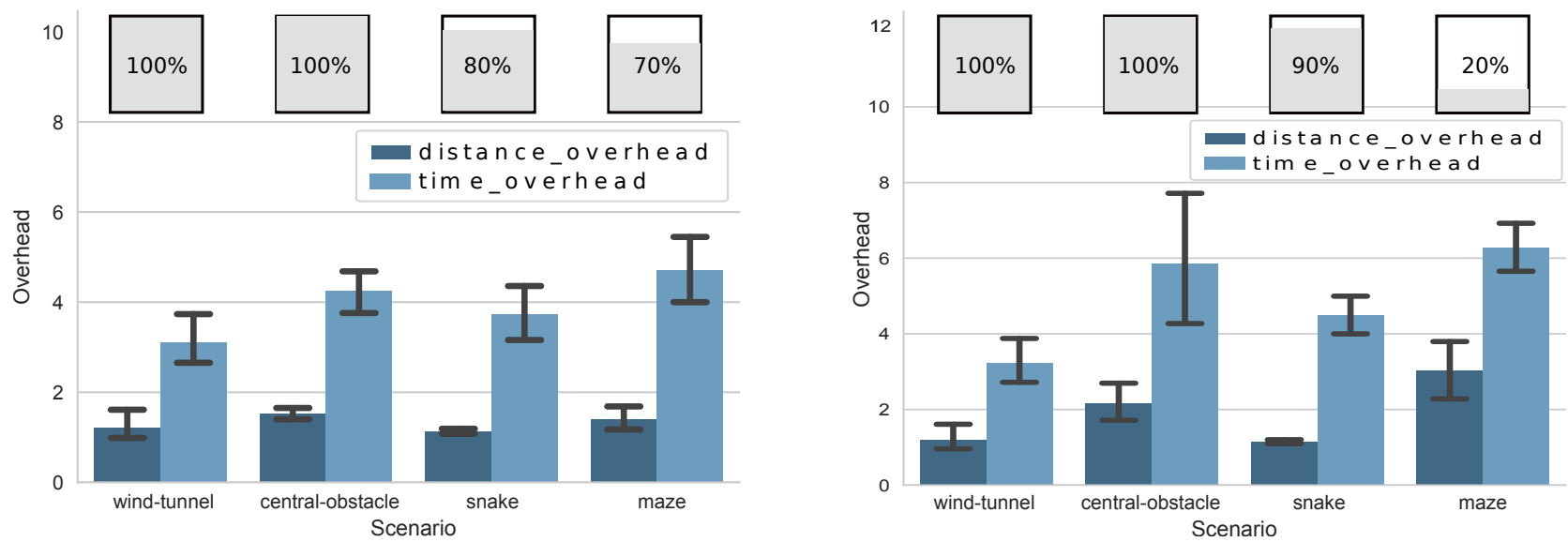

Fig. 4. Experimental results depicting the distance overhead, search-time overhead and success rate (gray boxes) for the four different scenarios under two different boundary wind conditions: $0.5 \mathrm{~m} / \mathrm{s}$ (left) and $1 \mathrm{~m} / \mathrm{s}$ (right).

by setting, at the boundary, wind flows of average strength $0.5 \mathrm{~m} / \mathrm{s}$ and $1 \mathrm{~m} / \mathrm{s}$, respectively. The latter leads to narrower and patchier plumes that also tend to rise when facing obstacles, being more challenging the detection and tracking of the plume. Results are summarized in Fig. 4. As can be seen, the distance and time overheads tend to increase when obstacles are present, while the success rate decreases. This performance drop is more noticeable, as expected, when considering high wind speeds.

Analyzing each scenario we can say that for the wind-tunnel (used as a reference) we obtain almost perfect values, being the only exception the time-overhead. The reason is due to the fact that, even for straight plumes, the algorithm requires the robot to stop\&measure in many occasions to get reliable values of the wind direction. The introduction of the central obstacle causes the plume to break into two narrower and patchier plumes to each side of the environment (see Fig. 1). The latter increases the travelled distance and moreover the search time, since losing the plume is relatively common. Yet, plume-recovery actions successfully work in this scenario as reported by the $100 \%$ success rate achieved under both wind flow conditions.

The snake scenario can be seen as a long corridor that repeatedly turns right and left. Despite the continuous changes of wind direction the algorithm seems to work relatively well as noticed in the small search-time overhead and high success rate. Due the limited navigational areas in this setup, the distance overhead is almost despicable. Yet, some runs failed to reach the source because the robot got stuck in a corner when moving upwind. Finally, the maze scenario, which in essence is very similar to the snake one, surprisingly drops the success rate to $20 \%$ when considering boundary wind flows of $1 \mathrm{~m} / \mathrm{s}$. This is due to the turbulent wind flows that make the plume to rise and get too close to the walls, being very difficult to be detected by the sensors carried by the robot. The latter causes the robot to continuously execute plume recovery actions (reason for the increased distance and time overheads), while not being able to reach the source in most runs.

\section{CONCLUSIONS}

In this work we have presented an evaluation of a plume tracking algorithm under turbulent wind flow conditions and four challenging scenarios. Results confirm that obstacles induce a performance penalty both because of the path constraints and the turbulence downwind the obstacles. The latter causes the plume to become more peaky and therefore loosing the plume is very common, being necessary to have appropriate plume-recovery mechanisms.

Potential future research directions include studies with more and diverse scenarios, and comparison between different gas source localization algorithms.

\section{REFERENCES}

[1] G. Kowadlo and R. A. Russell, "Robot odor localization: a taxonomy and survey," The International Journal of Robotics Research, vol. 27, no. 8, pp. 869-894, 2008.

[2] A. Lilienthal and T. Duckett, "Experimental analysis of smelling braitenberg vehicles," in IEEE International Conference on Advanced Robotics (ICAR), 2003, pp. 375-380.

[3] L. Marques, U. Nunes, and A. T. de Almeida, "Olfaction-based mobile robot navigation," Thin Solid Films, vol. 418, no. 1, pp. 51-58, 2002.

[4] J. Monroy and J. Gonzalez-Jimenez, Towards Odor-Sensitive Mobile Robots. IGI Global, 2018, pp. 244-263.

[5] H. Ishida, K. Suetsugu, T. Nakamoto, and T. Moriizumi, "Study of autonomous mobile sensing system for localization of odor source using gas sensors and anemometric sensors," Sensors and Actuators A: Physical, vol. 45, no. 2, pp. 153 - 157, 1994.

[6] L. Marques, N. Almeida, and A. De Almeida, "Olfactory sensory system for odour-plume tracking and localization," in IEE Sensors, vol. 1, 2003, pp. 418-423.

[7] T. Lochmatter and A. Martinoli, "Tracking odor plumes in a laminar wind field with bio-inspired algorithms," in Experimental robotics. Springer, 2009, pp. 473-482.

[8] — " "Theoretical analysis of three bio-inspired plume tracking algorithms," in IEEE International Conference on Robotics and Automation (ICRA), 2009, pp. 2661-2668.

[9] J. Monroy, J. R. Ruiz-Sarmiento, F.-A. Moreno, F. Melendez-Fernandez, C. Galindo, and J. Gonzalez-Jimenez, "A semantic-based gas source localization with a mobile robot combining vision and chemical sensing," Sensors, vol. 18, no. 12, 2018.

[10] J. Monroy, V. Hernandez-Bennetts, H. Fan, A. Lilienthal, and J. Gonzalez-Jimenez, "GADEN: A 3D gas dispersion simulator for mobile robot olfaction in realistic environments," MDPI Sensors, vol. 17, no. 7: 1479, pp. 1-16, 2017.

[11] J. Monroy, J.-L. Blanco, and J. Gonzalez-Jimenez, "Time-variant gas distribution mapping with obstacle information," Autonomous Robots, vol. 40 , no. 1 , pp. 1-16, 2016. 